\section{(6) OPEN ACCESS}

\title{
Prevalence of HIV and other sexually transmitted infections and factors associated with syphilis among female sex workers in Panama
}

\author{
Shilpa Hakre, ${ }^{1}$ Griselda Arteaga, ${ }^{2}$ Aurelio E Núñez, ${ }^{3}$ Christian T Bautista, ${ }^{4}$ \\ Aimee Bolen, ${ }^{1}$ Maria Villarroel, ${ }^{1}$ Sheila A Peel, ${ }^{5}$ Gabriela Paz-Bailey, ${ }^{6}$ Paul T Scott, ${ }^{5}$ \\ Juan M Pascale, ${ }^{2}$ the Panama HIV EPI Group
}

\begin{abstract}
'Department of Epidemiology and Threat Assessment, United States Military HIV Research Program, Henry M. Jackson Foundation for the Advancement of Military Medicine, Bethesda, Maryland, USA

${ }^{2}$ Genómica y Proteómica, Instituto Conmemorativo Gorgas para Estudios de la Salud, Panama, Panama ${ }^{3}$ National HIV/AIDS Control Program, Ministry of Health, Panama, Panama

${ }^{4}$ Department of Preventive Medicine, Walter Reed Army Institute of Research, Silver Spring, Maryland, USA ${ }^{5}$ United States Military HIV Research Program, Walter Reed Army Institute of Research, Rockville, Maryland, USA ${ }^{6}$ Centro de Estudios en Salud, Del Valle University of Guatemala, Guatemala, Guatemala
\end{abstract}

Correspondence to Dr Shilpa Hakre, Department of Epidemiology and Threat Assessment, US Military HIV Research Program, 6720-A Rockledge Drive, Suite 400, Bethesda, MD 20817, USA; shakre@hivresearch.org

Received 28 February 2012 Revised 6 August 2012 Accepted 8 August 2012 Published Online First 21 September 2012

\section{ABSTRACT}

Objectives Biological and behavioural surveillance of HIV and sexually transmitted infections (STIS) among populations at highest risk have been used to monitor trends in prevalence and in risk behaviours. Sex work in Panama is regulated through registration with the Social Hygiene Programme, Ministry of Health. We estimated prevalence of HIV and STIs, and factors associated with active syphilis among female sex workers (FSWs).

Methods A cross-sectional study using venue-based, time-space sampling was conducted among FSWs in Panama from 2009 to 2010. FSWs were interviewed about sociodemographic characteristics, sexual risk behaviour, health history and drug use using an anonymous structured questionnaire. Blood was collected for serological testing of HIV and other STIs. Factors associated with active syphilis were studied using logistic regression analysis.

Results The overall HIV-1 prevalence of $0.7 \%$ varied by FSW category; $1.6 \%$ in 379 unregistered, and $0.2 \%$ in 620 registered FSWs. Overall prevalence (and 95\% CI) of STIs were: syphilis antibody, $3.8 \%$ (2.7\% to $5.2 \%)$; herpes simplex virus type 2 antibody (anti-HSV-2), 74.2\% (71.4\% to $76.9 \%)$; hepatitis B surface antigen, $0.6 \%$ ( $0.2 \%$ to $1.3 \%)$; hepatitis B core antibody, $8.7 \%(7.0 \%$ to $10.6 \%)$; and hepatitis C antibody, $0.2 \%(0.0 \%$ to $0.7 \%)$. In multivariate analysis, registration (adjusted OR (AOR) $=0.35 ; 95 \% \mathrm{Cl} 0.16$ to 0.74 ), having a history of STI (AOR $=2.37 ; 95 \% \mathrm{Cl} 1.01$ to 5.58), forced sex $(\mathrm{AOR}=2.47 ; 95 \% \mathrm{Cl} 1.11$ to 5.48$)$, and anti-HSV-2 $(\mathrm{AOR}=10.05 ; 95 \% \mathrm{Cl} 1.36$ to 74.38$)$ were associated with active syphilis

Conclusions Although HIV prevalence is low among FSWs in Panama, unregistered FSWs bear a higher burden of HIV and STIs than registered FSWs. Programmes aimed at overcoming obstacles to registration, and HIV, STI and harm reduction among unregistered FSWs is warranted to prevent HIV transmission, and to improve their sexual and reproductive health.

\section{INTRODUCTION}

Central and South America and the Caribbean together accounted for the second highest adult HIV prevalence in the world in 2009. ${ }^{1}$ The overall estimated adult HIV prevalence in Central and South America is $0.5 \%$ and ranges from $2.3 \%$ in Belize, to at least $1 \%$ in Guyana and Suriname, and $0.9 \%$ in Panama. ${ }^{2}$ In most of these countries, the HIV epidemic is concentrated in populations at highest risk: men who have sex with men (MSM) and commercial sex workers. ${ }^{2}$

In countries with concentrated epidemics, the second-generation HIV surveillance approach among populations at highest risk provides valuable data to monitor trends in prevalence of infection, and the risk behaviours driving these trends which, together, inform the evaluation of existing national AIDS control programmes. ${ }^{3}$ Some of the key methods in second-generation HIV surveillance include repeated cross-sectional HIV sentinel surveillance combined with behavioural and sexually transmitted infections (STIs) surveillance.

An estimated 3105 women worked as sex workers in 2010 in Panama (Office of Registration and Health Statistics, Ministry of Health, Panama); 98\% were under 50 years of age. The Ministry of Health $(\mathrm{MoH})$ has regulated work at adult entertainment venues by registration through the Social Hygiene Programme, introduced in Panama in 1918. ${ }^{4}$ The penalty for non-registration is a fine of US $\$ 500$ levied on the owner of the venue employing the sex worker. Registration costs, US $\$ 100-150$ every 3 months, which includes costs of a mandatory photo-identification card, mandatory testing (weekly, for bacterial STIs; quarterly, for HIV and syphilis) at $\mathrm{MoH}$ health centres; a positive HIV result leads to revocation of registration and deportation for non-Panamanian female sex workers (FSWs) with a work visa. HIV testing is available at laboratories in all health districts. Treatment and care for HIV-positive persons are an individual's right under Panamanian law.

As a transit point between Central and South America, and between the Atlantic and Pacific Oceans, Panama is vulnerable to HIV and STIs prevalent in its migrant population. ${ }^{5}$ Women and children from neighbouring countries have been trafficked into the sex trade both within and through Panama. ${ }^{6}$ In-depth interviews of various at-risk populations in transit areas in Central America identified the MSM population in Panama as having considerable contact with mobile populations. ${ }^{5}$ Continued HIV and STI surveillance is necessary to guide prevention programmes among high-risk groups to prevent the spread of the HIV epidemic from such groups serving as a bridge to the general population. The last HIV and STI 
second-generation surveillance conducted in 2003 in Panama and Colón, two port cities, indicated a HIV prevalence of $2 \%$ among FSWs. ${ }^{7}$ We report results from a repeat HIV and STI biobehavioural survey conducted among FSWs in Panama. The objectives were to determine the prevalence of HIV, active syphilis and other viral STIs, and to assess factors associated with active syphilis.

\section{MATERIALS AND METHODS Study design and study population}

Panama's administrative division consists of nine provinces and three indigenous territories. A cross-sectional biobehavioural survey among FSWs was conducted from October 2009 to May 2011 in eight provinces: Panama, Colón, Coclé, Herrera, Los Santos, Veraguas, Chiriquí and Bocas del Toro; the Darien region was excluded for security reasons. Herrera and Los Santos were combined for recruitment and analysis; the two provinces share geographic boundaries and considerable intermigration for health and other services. Accounting for difficulty in accessing this hard-to-reach population, ${ }^{8}$ an enrolment of 1200 participants, $25 \%$ unregistered/street and $75 \%$ registered, was the estimated target sample size for $80 \%$ power, HIV prevalence of $1 \%$, and prevalence precision of $1.3 \%$. Criteria for enrolment were: (1) female gender; (2) age 18 years or older; (3) self-identified sex worker and (4) willingness to provide written informed consent.

\section{Sampling methodology}

FSWs were recruited using venue-based, time-space sampling (TSS). ${ }^{9}$ Activities included: (1) formative research to identify venues and time to recruit FSWs; (2) development of sampling frames of eligible venues and day-time periods; (3) random selection of venues and day-time periods; (4) recruitment and referral and (5) interviewing, and testing at study sites.

Survey staff identified venues for registered and unregistered FSWs or 'clandestinas' by contacting non-governmental organisations (NGOs) who had worked with FSWs in the year before the survey began, and key informants who worked at FSW work locations for 3 months or more. In two visits or more, survey staff estimated the FSW population size at various daytime and night-time hours at all identified venues (bars, taverns, night clubs, brothels, parks, city blocks, health clinics, street corners and pimps). Venues were chosen based on accessibility and overall interest in study participation. At venues with an estimated FSW population of 8-20, all women were invited to participate, whereas at venues with 20-plus FSWs, women were invited randomly with a simple coin toss.

At 67 venues, women self-identifying as registered FSWs were informed of the survey, invited to participate, and handed a coded pocket-sized calendar which indicated $\mathrm{MoH}$ health centres' staff availability. The code indicated the site and month of recruitment and an invitation number. At an additional 54 venues, and through two pimps in one province, women self-identifying as unregistered FSWs were handed a second coded invitation card to recruitment dinners for enrolment and blood collection.

All interested and eligible women with invitation cards were enrolled in the study at either $\mathrm{MoH}$ health centres or recruitment dinners. Trained female interviewers administered an anonymous structured questionnaire after obtaining informed consent from participants. A rapid HIV test was performed on-site. Participants' questionnaires and blood samples were assigned unique codes. Survey and $\mathrm{MoH}$ staff conducted pretest counselling before HIV-1 rapid testing of participants. Participants were asked to return in 2 weeks for the test results-health centres for registered FSWs or NGOs for unregistered-FSWs - and if positive, were provided post-test counselling and referred to $\mathrm{MoH}$ clinics for free medical care, treatment and contact tracing. All participants were given an incentive - a grocery store gift card or a US $\$ 15.00$ cosmetic kit.

\section{Ethical considerations}

The study was approved by two institutional review boards: Instituto Conmemorativo Gorgas para Estudios de la Salud (ICGES, Panama), Walter Reed Army Institute of Research (WRAIR, USA). Only the Panama study team had access to informed consent forms.

\section{Data collection}

Staff conducted face-to-face interviews with a structured questionnaire which elicited information about sociodemographic characteristics, sexual risk behaviour, health history and alcohol and drug use. Sociodemographic variables included age, nationality, education level, current marital status, race, current monthly income and employment, dependents and children, current residence and registration card on-person. Sexual risk behaviour history included age of sexual debut with a male, duration as sex worker, type of and number of sexual contacts in the past 6 months, condom use and sex work locations. Health history included contraceptive use, STI history, transfusion, tattoos, abortions, reasons for abortion, HIV testing and last time of testing, sexual contact during menstrual period, forced sex and whether a regular partner had HIV/AIDS. Alcohol and drug use questions included lifetime drug or alcohol use, frequency and timing of alcohol use.

\section{Laboratory procedures}

Rapid HIV testing (US Food and Drug Administration-approved HIV 1/2 STAT PAK, Assay Chembio Diagnostic System, Inc, Medford, New York, USA) via fingerstick was performed at recruitment sites. In addition, $10 \mathrm{ml}$ of blood via peripheral venipuncture was collected, centrifuged and separated into aliquots on-site, and transported to the study laboratory for storage at $4^{\circ} \mathrm{C}$.

Samples were evaluated for HIV (antibody to HIV (anti-HIV) and p24 antigen, AxSYM HIV Ag/Ab Combo), antibody to hepatitis $\mathrm{C}$ virus ((anti-HCV), AxSYM HCV V.3.0), hepatitis B surface antigen ((HBsAg), AxSYM HBsAg (V2)), antibody to hepatitis B core antigen ((anti-HBc), AxSYM CORE), antibody to herpes simplex type 2 virus ((anti-HSV2), ELISA, HerpeSelec-2, Focus Diagnostics, Cypress, California, USA), and syphilis (rapid plasma reagin (RPR), Arlington Scientific, Inc, Springville, Utah, USA). HIV, hepatitis B virus (HBV), and HCV testing was performed on an AxSYM analyser (Abbott, Wiesbaden, Germany) using microparticle enzyme immunoassay technology.

All initially reactive serological AxSYM tests were repeated in duplicate; HIV samples were repeated in duplicate irrespective of initial results. Positive screening results were confirmed for HIV (GS HIV-1 Western Blot, BioRad Laboratories, Redmond, Washington, USA), HCV (recombinant immunoblot assay (RIBA), Chiron RIBA HCV 3.0, Emeryville, California, USA) and syphilis (Treponema pallidum haemagglutination (TPHA), Arlington Scientific, Inc). Participants with laboratoryconfirmed syphilis-positive results, defined as RPR and TPHA positivity, were considered to have active syphilis infection. ${ }^{10}$ (Henceforth, the term syphilis denotes active syphilis). 


\section{Analysis}

Prevalence was estimated for HIV, HBV, HCV, HSV-2 and syphilis. Exact binomial or binomial $95 \%$ CIs were calculated for prevalence estimates. Characteristics were compared by registration status using $\chi^{2}$ or Fisher's exact test for categorical variables and t test or Kruskal-Wallis test for continuous variables. Prevalence ORs were estimated in a univariate analysis. Characteristics associated with syphilis in a univariate analysis $(p<0.20)$ were evaluated for correlation and entered in a multivariate logistic regression model. Characteristics that were not statistically significant $(p<0.05)$ in the multivariate model were removed in a stepwise manner, and independent association with syphilis was assessed after adjustment for other variables retained in the model. Double data entry for all questionnaire and laboratory data were conducted at the ICGES. All data management and analyses were conducted using SAS V.9.2 (SAS Institute Inc, Cary, North Carolina, USA).

\section{RESULTS}

\section{Study population}

Of 1250 invitations issued, 1000 (80\%) FSWs participated; one participant consented only to the questionnaire; $62 \%$ selfidentified as registered FSWs of whom $86 \%$ had a registration card at interview. Of 999 participants, 79\% registered and 61\% unregistered FSWs returned for the test results. The majority of FSWs (46\%) were recruited from Panama. FSWs averaged 29.4 years in age ( $\mathrm{SD} \pm 8.2$ years) and most were single $(74 \%)$.

\section{Prevalence of HIV, syphilis and other STIs}

The overall HIV prevalence among FSWs was $0.7 \%$ (95\% CI 0.3 to $1.4 \%$ ); unregistered FSWs had a higher prevalence than registered FSWs (1.6\% (95\% CI 0.6 to $3.4 \%$ ), $0.2 \%$ (95\% CI 0.0 to $0.9 \%$ ), respectively; $p=0.014$; figure 1$)$. HIV prevalence varied by province among participants (Bocas Del Toro: $2.1 \%$, 95\% CI 0.3 to $7.4 \%$; Coclé, Chiriquí, Herrera and Los Santos, and Veraguas: $0.0 \%$, 95\% CIs 0.0 to $12.3 \%$ ).

Among all participants, 3.8\% (95\% CI 2.7 to 5.2) were infected with syphilis (figure 1). Syphilis was higher among unregistered FSWs $(6.6 \%, 95 \%$ CI 4.3 to $9.6 \%$ ) than in registered FSWs $(2.1 \%$, 95\% CI 1.1 to $3.6 \%, p=0.0003)$.The prevalence of syphilis varied by province ranging from $8.4 \%$ (Bocas Del Toro, $95 \%$ CI 3.7 to $15.9 \%$ ) to 1.6-2.6\% (Coclé, Herrera and Los Santos, Colón, and Chiriquí, 95\% CIs 0.0 to 10.3\%).

The overall prevalence of $\mathrm{HSV}-2$, anti-HBc, and $\mathrm{HCV}$ were $74.2 \%$ (95\% CI 71.4 to $76.9 \%$ ), $8.7 \%$ (95\% CI 7.0 to $10.6 \%$ ), and $0.2 \%$ (95\% CI 0.0 to $0.7 \%$ ), respectively. Of the six $(0.6 \%, 95 \%$ CI 0.2 to $1.3 \%$ ) HBsAg-positive participants, five were anti-HBc positive. One participant was coinfected with HIV and syphilis.

\section{Comparison between registered and unregistered FSWs}

In bivariate analysis, registered and unregistered FSWs differed significantly by nationality, education levels, income, number of dependents, number of children, sexual risk behaviour, health history and drug use $(p<0.01$, table 1$)$. Unregistered FSWs were mostly Panamanian (89\%) with lower levels of education $(68 \%$ incomplete secondary or less vs $45 \%$ ), more children (mean: 2.5 vs

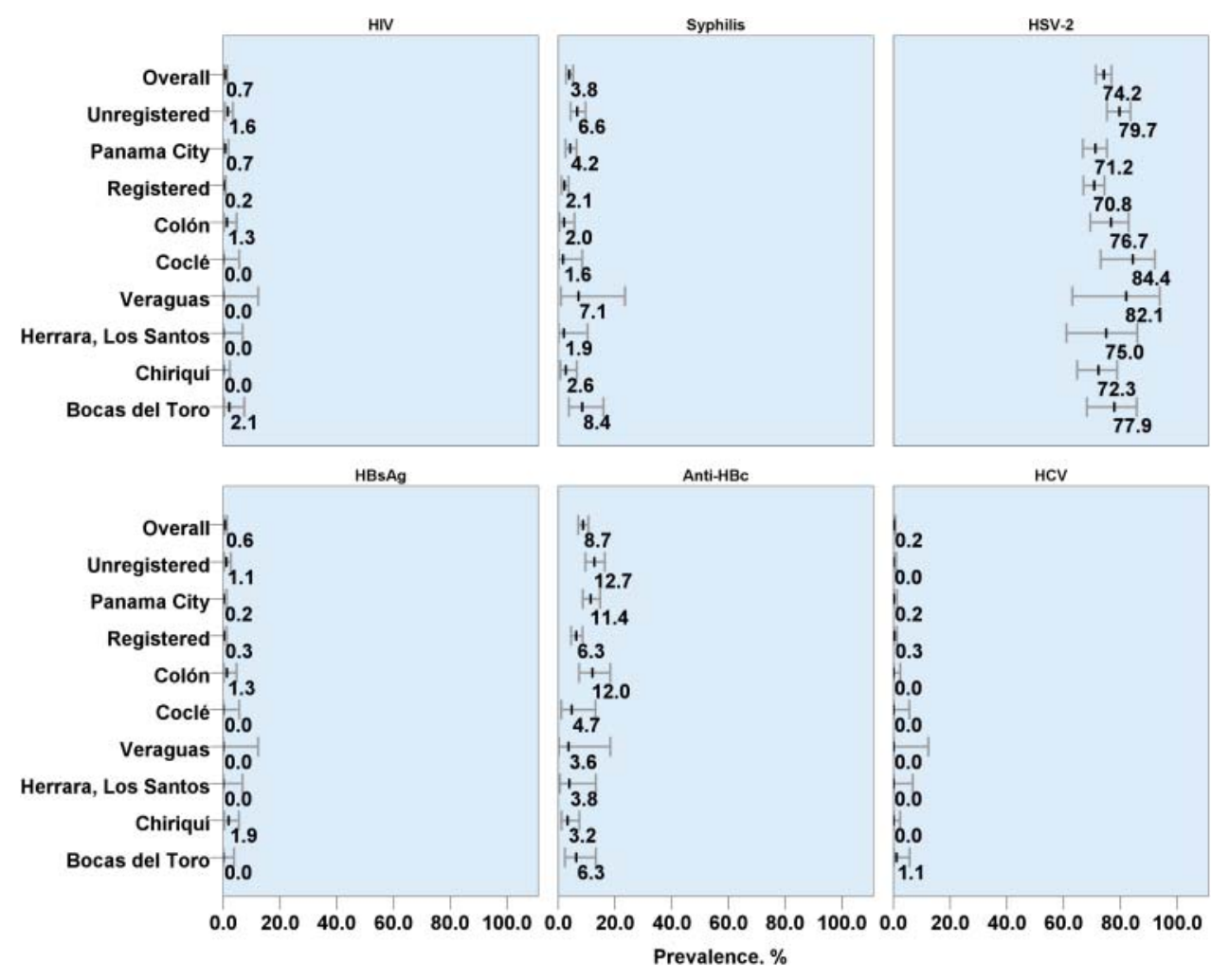

Figure 1 Seroprevalence and 95\% confidence intervals of HIV, syphilis and other viral sexually transmitted diseases among 999 female sex workers from eight provinces, Panama, October 2009-May 2011. Female sex workers (FSWs) category: unregistered FSWs $n=379$, registered FSWs $n=620$; Province: Panama $n=455$, Coclé $n=64$, Colón $n=150$, Chiriquí $n=155$, Herrera and Los Santos $n=52$, Bocas del Toro $n=95$, Veraguas $n=28$. Of 999 FSWs, 0.4\% had HCV indeterminate results $(n=4$; unregistered FSWs $n=3$, registered FSWs $n=1)$. Anti-HBc seroprevalence varied significantly by site $(p=0.01)$ and registration status $(p=0.001)$; HIV $(p=0.014)$, syphilis $(p<0.0005)$, anti-Hbc, and HSV-2 $(p=0.001)$ varied significantly by registration status. 
Table 1 Characteristics of female sex workers (FSWs) and comparisons between unregistered and registered FSWs recruited from eight provinces, Panama, 0ctober 2009-May 2011

\begin{tabular}{|c|c|c|c|c|c|c|c|c|}
\hline \multirow[b]{2}{*}{ Category } & \multirow[b]{2}{*}{ Characteristic } & \multicolumn{2}{|c|}{ Overall $(n=999)$} & \multicolumn{2}{|c|}{ Unregistered $(n=379)$} & \multicolumn{2}{|c|}{ Registered $(n=620)$} & \multirow[b]{2}{*}{ p Value } \\
\hline & & $n /$ total & $\%$ & n/total & $\%$ & n/total & $\%$ & \\
\hline \multirow[t]{31}{*}{ Sociodemographics } & Province & & & & & & & \\
\hline & Panama & 455 & 46 & 157 & 41 & 298 & 48 & \\
\hline & Coclé & 64 & 6 & 15 & 4 & 49 & 8 & \\
\hline & Colón & 150 & 15 & 57 & 15 & 93 & 15 & \\
\hline & Chiriquí & 155 & 15 & 66 & 17 & 89 & 14 & \\
\hline & Herrera, Los Santos & 52 & 5 & 0 & 0 & 52 & 8 & \\
\hline & Bocas del Toro & 95 & 9 & 65 & 17 & 30 & 5 & \\
\hline & Veraguas & 28 & 3 & 19 & 5 & 9 & 1 & \\
\hline & Age, mean (range) & 29.4 & $(18-61)$ & 29.4 & $(18-61)$ & 29.5 & $(18-57)$ & 0.8193 \\
\hline & 24 or less & $320 / 999$ & 32 & $149 / 379$ & 39 & $171 / 620$ & 28 & \\
\hline & $25-29$ & $261 / 999$ & 26 & $69 / 379$ & 18 & $192 / 620$ & 31 & \\
\hline & $30-39$ & $271 / 999$ & 27 & $90 / 379$ & 24 & $181 / 620$ & 29 & \\
\hline & $40+$ & $139 / 999$ & 14 & $70 / 379$ & 18 & $69 / 620$ & 11 & \\
\hline & Nationality & & & & & & & $<0.0001$ \\
\hline & Panama & $487 / 987$ & 49 & $338 / 377$ & 89 & $149 / 610$ & 24 & \\
\hline & Colombia & $412 / 987$ & 41 & $12 / 377$ & 3 & $400 / 610$ & 65 & \\
\hline & Dominican Republic & $67 / 987$ & 7 & $19 / 377$ & 5 & $48 / 610$ & 8 & \\
\hline & Other* & $21 / 987$ & 2 & $8 / 377$ & 2 & $13 / 610$ & 2 & \\
\hline & Education & & & & & & & $<0.0001$ \\
\hline & None or primary school or less & $146 / 992$ & 15 & $58 / 378$ & 15 & $88 / 614$ & 14 & \\
\hline & Incomplete secondary school & $390 / 992$ & 39 & $200 / 378$ & 53 & $190 / 614$ & 31 & \\
\hline & Complete secondary/ technical school or university & $456 / 992$ & 46 & $120 / 378$ & 32 & $336 / 614$ & 54 & \\
\hline & Marital status & & & & & & & 0.0427 \\
\hline & Single & $741 / 990$ & 74 & $268 / 376$ & 71 & $473 / 614$ & 76 & \\
\hline & Othert & $249 / 990$ & 25 & $108 / 376$ & 29 & $141 / 614$ & 23 & \\
\hline & Other dependents & $672 / 965$ & 70 & $205 / 363$ & 56 & $467 / 602$ & 77 & $<0.0001$ \\
\hline & Mean number of children (range) & 2 & $(0-11)$ & 2.5 & $(0-11)$ & 1.7 & $(0-11)$ & $<0.0001$ \\
\hline & Median income, USD (IOR) & 485 & $(250-800)$ & 250 & $(120-430)$ & 500 & $(350-1000)$ & $<0.0001$ \\
\hline & Work location $\ddagger$ & & & & & & & $<0.0001$ \\
\hline & Premises & $675 / 832$ & 68 & $140 / 296$ & 37 & $535 / 536$ & 86 & \\
\hline & Street & $157 / 832$ & 16 & $156 / 296$ & 41 & $1 / 536$ & 0 & \\
\hline \multicolumn{9}{|l|}{ Sexual risk behaviour } \\
\hline & Mean age at sexual debut with male, years (range) & 15.9 & $(7-27)$ & 15.6 & $(7-25)$ & 16.1 & $(9-27)$ & 0.0007 \\
\hline & Median number of males, sexual contact in past 6 months (IOR) & 20 & $(5-100)$ & 10 & $(4-30)$ & 40 & $(6-180)$ & $<0.0001$ \\
\hline & Median number of years worked as sex worker (IOR) & 2 & $(0.6-5)$ & 3 & $(1-6)$ & 1 & $(0.5-3)$ & $<0.0001$ \\
\hline & Client & & & & & & & \\
\hline & Vaginal sex & $864 / 895$ & 97 & $335 / 339$ & 99 & $529 / 556$ & 95 & 0.0035 \\
\hline & Oral sex & $517 / 882$ & 59 & $177 / 337$ & 53 & $340 / 545$ & 62 & 0.0039 \\
\hline & Anal sex & $130 / 883$ & 15 & $76 / 339$ & 22 & $54 / 544$ & 10 & $<0.0001$ \\
\hline & Not regular partner & & & & & & & \\
\hline & Vaginal sex & $852 / 968$ & 88 & $327 / 363$ & 90 & $525 / 602$ & 87 & 0.1787 \\
\hline
\end{tabular}


Table 1 Continued

\begin{tabular}{|c|c|c|c|c|c|c|c|c|}
\hline \multirow[b]{2}{*}{ Category } & \multirow[b]{2}{*}{ Characteristic } & \multicolumn{2}{|c|}{ Overall $(\mathrm{n}=999)$} & \multicolumn{2}{|c|}{ Unregistered $(n=379)$} & \multicolumn{2}{|c|}{ Registered $(n=620)$} & \multirow[b]{2}{*}{ p Value } \\
\hline & & $\mathrm{n} /$ total & $\%$ & $\mathrm{n} /$ total & $\%$ & $\mathrm{n} /$ total & $\%$ & \\
\hline & Oral sex & $504 / 950$ & 53 & $173 / 363$ & 48 & $331 / 587$ & 56 & 0.0088 \\
\hline & Anal sex & 141/935 & 15 & $78 / 360$ & 22 & $63 / 575$ & 11 & $<0.0001$ \\
\hline & Current regular partner & & & & & & & \\
\hline & Vaginal sex & $502 / 735$ & 68 & 201/282 & 71 & $301 / 453$ & 66 & 0.1711 \\
\hline & Oral sex & $339 / 697$ & 49 & 114/266 & 43 & $225 / 431$ & 52 & 0.0165 \\
\hline & Anal sex & 158/691 & 23 & 79/262 & 30 & 79/429 & 18 & 0.0004 \\
\hline & Alcohol/drug use by regular partner before/during sex, sometimes-always & 158/447 & 35 & 75/188 & 40 & 83/259 & 32 & 0.0008 \\
\hline & Sex during menstruation & $514 / 991$ & 52 & 177/377 & 47 & $337 / 614$ & 55 & 0.0152 \\
\hline & Forced sex & 132/987 & 13 & 62/374 & 17 & 70/613 & 11 & 0.0209 \\
\hline & Sexual contact with an injection drug user & 136/727 & 19 & $67 / 289$ & 23 & 69/438 & 16 & 0.0119 \\
\hline & Sexual contact with male sex workers & 66/955 & 7 & $41 / 365$ & 11 & $25 / 590$ & 4 & $<0.0001$ \\
\hline & Sex work in another country & $178 / 890$ & 20 & $16 / 333$ & 5 & 162/557 & 29 & $<0.0001$ \\
\hline & Currently travel to another country for sex work & $76 / 879$ & 9 & $11 / 331$ & 3 & $65 / 548$ & 12 & $<0.0001$ \\
\hline & Sexual contact ever in another country & 687/967 & 71 & 210/367 & 57 & $477 / 600$ & 80 & $<0.0001$ \\
\hline & Sexual contact on travel in past 6 months, non regular partner & 127/961 & 13 & 14/363 & 4 & $113 / 598$ & 19 & $<0.0001$ \\
\hline & Regular client construction worker & $346 / 999$ & 35 & $156 / 379$ & 41 & 190/620 & 31 & 0.0007 \\
\hline & Regular client in military & 79/999 & 8 & 24/379 & 6 & $55 / 620$ & 9 & 0.1491 \\
\hline & Regular client a foreigner & 292/999 & 29 & $101 / 379$ & 27 & $191 / 620$ & 31 & 0.1609 \\
\hline \multicolumn{9}{|c|}{ Health history } \\
\hline & Mean days since ever HIV tested (range) & 211.6 & $(1-6496)$ & 463.7 & $(1-6496)$ & 114.7 & $(1-3090)$ & $<0.0001$ \\
\hline & Transfusions & 94/968 & 10 & 47/370 & 13 & $47 / 598$ & 8 & 0.0134 \\
\hline & Medications by injections & 637/976 & 65 & $210 / 367$ & 57 & $427 / 609$ & 70 & $<0.0001$ \\
\hline & Have tattoos & 409/994 & 41 & 174/378 & 48 & $235 / 616$ & 38 & 0.0142 \\
\hline & Have piercings & 204/992 & 21 & 61/377 & 16 & $143 / 615$ & 23 & 0.0075 \\
\hline \multicolumn{9}{|l|}{ Drug use } \\
\hline & Never used drugs & 737/999 & 74 & 257/379 & 68 & $480 / 620$ & 77 & 0.0008 \\
\hline & Used marijuana & 152/999 & 15 & 77/379 & 20 & $75 / 620$ & 12 & 0.0004 \\
\hline & Used cocaine & 93/999 & 9 & $43 / 379$ & 11 & $50 / 620$ & 8 & 0.0833 \\
\hline
\end{tabular}

tMarried, divorced, widowed, separated, living with someone.

t'Premises' included work locations bar/taberna, discotheque, whorehouse, motel, house/home, saunas/masseuse, gym, public bath, theatres, and night clubs. 
1.7), and lower income (median USD: 250 vs 500 ) than registered FSWs. Registered FSWs were predominantly Colombian (65\%).

Unregistered FSWs reported fewer sexual contacts in the past 6 months (median number of males, 10 (IOR: 4-30) vs 40 (IOR: 6-180)), were younger at sexual debut with a male (mean years: 15.6 vs 16.1), had worked longer as sex workers (median years: 3 vs 1 ), and more frequently reported engaging in anal sex with clients $(22 \%)$, regular $(30 \%)$ and non-regular $(22 \%)$ partners than registered FSWs $(10 \%, 18 \%$, 11\%, respectively, table 1$)$. Unregistered FSWs more frequently reported having had sexual contact with an injection drug user (23\% vs $16 \%$ ), male sex workers (11\% vs $7 \%$ ), using marijuana $(20 \%$ vs $12 \%)$, and having a regular partner who sometimes or always used alcohol or drugs before sex (40\% vs 32\%). Unregistered FSWs reported having had a HIV test less recently than registered FSWs (mean days since last HIV test, 463.7 vs 114.7). Unregistered FSWs more frequently reported never or inconsistently using condoms for anal sex with clients ( $27 \%$ vs $10 \%, p=0.02)$, and for vaginal sex with clients $(19 \%$ vs $4 \%, \mathrm{p}<0.0001)$ and non-regular partners $(20 \%$ vs $8 \%, p<0.0001$, figure 2$)$. Both unregistered and registered FSWs reported never or inconsistently using condoms with regular partners $(66 \%-80 \%$, figure 2$)$.

\section{Factors associated with syphilis}

In the univariate analysis, factors associated with syphilis were: registration, age of 40 years or older, having Panamanian nationality, being of African descent, having lower levels of education, working on the street, anti-HBc or HSV-2 positivity, forced sex, having clients who worked in construction or transportation or who were in the military, anal sex with a nonregular partner, inconsistently or never using condoms for vaginal sex, sexual contact with foreigners, having a history of STI and never using drugs $(p<0.05$, table 2$)$.

All factors associated with syphilis in the univariate analysis $(p<0.20$, table 2$)$ were entered into a multivariate model. Factors not retained in the final model $(p<0.05)$-nationality, education level, race, work location, sexual contact with foreigners, sex work in another country and contraceptive usewere highly correlated with registration status $(p<0.0001$, data not shown). In the final model, four factors showed independent association with syphilis: registration (adjusted OR (AOR) $=0.35$; $95 \%$ CI 0.16 to 0.74$)$, having a STI history $(\mathrm{AOR}=2.37$; $95 \% \mathrm{CI} 1.01$ to 5.58$)$, forced sex $(\mathrm{AOR}=2.47 ; 95 \% \mathrm{CI} 1.11$ to 5.48), and having HSV-2 antibody ( $\mathrm{AOR}=10.05$; 95\% CI 1.36 to 74.38 ) (table 2).

\section{DISCUSSION}

This biobehavioural survey was conducted among FSWs in eight provinces of Panama to monitor the prevalence of HIV, other viral STIs, syphilis among women at high risk for STIs, and to assess factors associated with syphilis. The overall HIV prevalence while low differed markedly by registration status. The

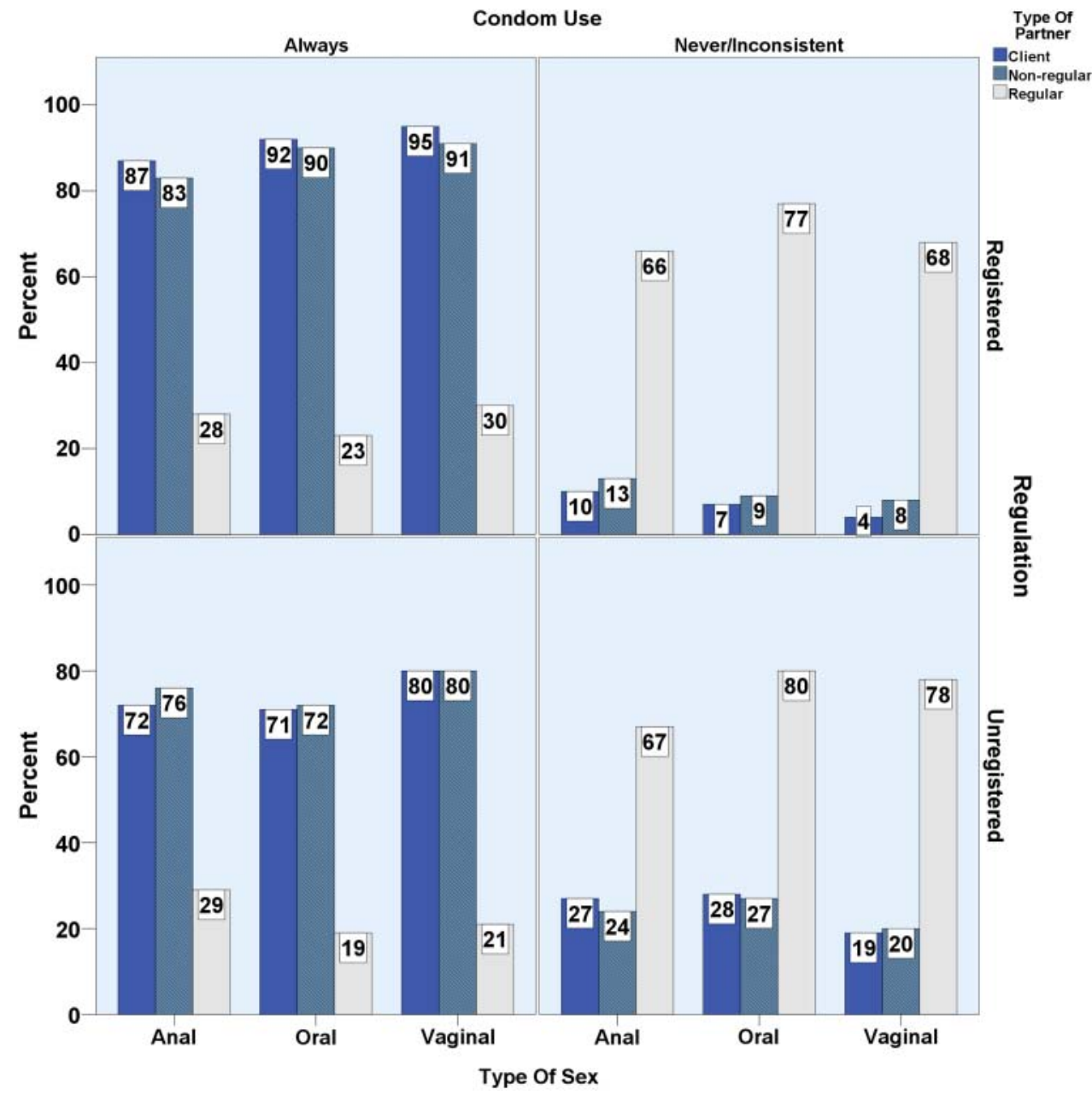

Figure 2 Percent of condom use reported by registered and unregistered female sex workers for sexual partners. 
Epidemiology

Table 2 Factors associated with syphilis in female sex workers from eight provinces in Panama, October 2009-May 2011

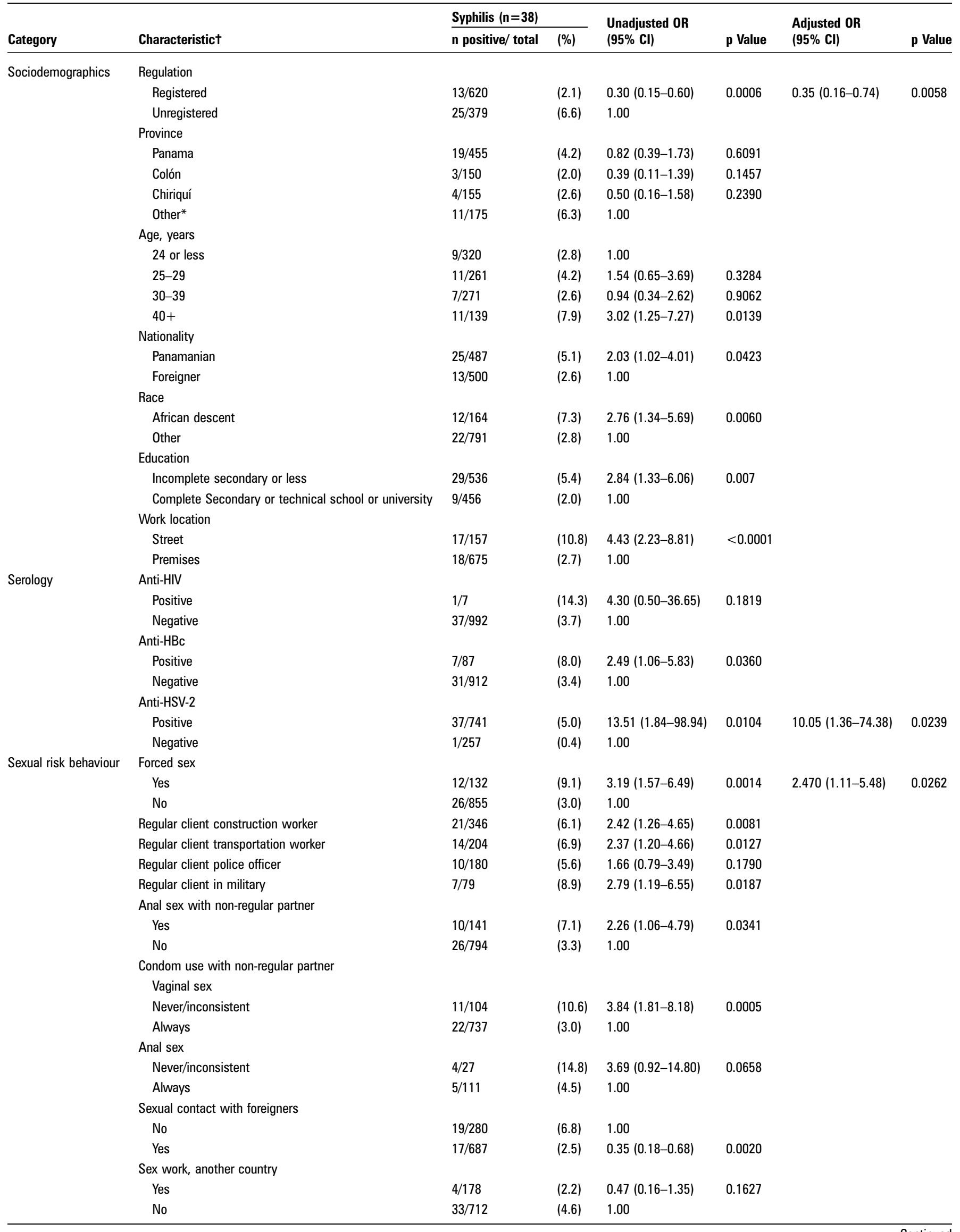


Table 2 Continued

\begin{tabular}{|c|c|c|c|c|c|c|c|}
\hline \multirow[b]{2}{*}{ Category } & \multirow[b]{2}{*}{ Characteristic $†$} & \multicolumn{2}{|l|}{ Syphilis $(n=38)$} & \multirow{2}{*}{$\begin{array}{l}\text { Unadjusted OR } \\
\text { (95\% CI) }\end{array}$} & \multirow[b]{2}{*}{ p Value } & \multirow{2}{*}{$\begin{array}{l}\text { Adjusted OR } \\
\text { (95\% Cl) }\end{array}$} & \multirow[b]{2}{*}{ p Value } \\
\hline & & n positive/ total & $(\%)$ & & & & \\
\hline \multirow[t]{4}{*}{ Health history } & Sexually transmitted infection history & & & & & & \\
\hline & No & $29 / 888$ & (3.3) & 1.00 & & & \\
\hline & Use contraceptives & & & & & & \\
\hline & No & $15 / 285$ & (5.3) & $0.63(0.32-1.23)$ & 0.1768 & & \\
\hline Drug use & Used marijuana & $11 / 152$ & (7.2) & $2.37(1.15-4.89)$ & 0.0194 & & \\
\hline
\end{tabular}

*`Other' included Coclé, Herrera, Los Santos, Bocas del Toro, and Veraguas.

tOnly characteristics significant at $\mathrm{p}<0.20$ are shown and were considered in multivariate analysis.

difference by registration status was seen in STI prevalence and HIV risk. Furthermore, registration was protective for syphilis.

Among FSWs who participated in the survey, the overall seroprevalence of HIV, syphilis and HSV-2 were $0.7 \%, 3.8 \%$ and $74.2 \%$, respectively. In the two port cities, Panama and Colón, the HIV, syphilis and HSV-2 seroprevalences were $0.8 \%, 3.6 \%$ and $72.6 \%$, respectively. A multicentric, multicountry, crosssectional survey conducted in 2003 among FSWs, with $15 \%$ of 404 FSWs recruited from streets and parks in the two port cities, reported a HIV, syphilis and HSV-2 seroprevalences of $1.9 \%, 4.8 \%$ and $73.0 \%$, respectively. ${ }^{7}$ The HSV-2 seroprevalence reported in the 2003 study was the lowest among the five Central American countries. ${ }^{8}$ Panama ranked above average in the region in prioritising domestic resources to AIDS treatment and HIV prevention. ${ }^{11}$ While it is possible our findings are reflective of the HIV epidemic stabilising from 2001 to 2009 in Central and South America, and an increased public spending on HIV prevention and treatment programmes in the region, the difference in our findings from the previous study is possibly the result of differing sampling methodologies, TSS versus cluster sampling. ${ }^{2} 11$

As reported previously in Panama, the prevalence of HIV and STIs varied widely by sex worker registration status. Unregistered FSWs had an eightfold higher HIV prevalence than registered FSWs. Similarly for syphilis, unregistered FSWs had a threefold higher prevalence than registered FSWs.

Compared with registered FSWs, unregistered FSWs reported higher levels of HIV risk, were socioeconomically disadvantaged and predominantly Panamanian. Among other factors, unregistered FSWs reported higher frequency of anal sex, lower frequency of condom use for anal sex, more contact with high-risk groups like injection drug users and male sex workers, and longer duration since the last HIV test. Contact with highrisk groups and lower condom use indicate unregistered FSWs may serve as an active bridging group for HIV and STI transmissions. Significant differences in income, education, exposure to violence, drug use, STIs, HIV risk and nationality by sex worker category have been reported in other countries. ${ }^{12-14}$ In Tijuana, Mexico, unregistered FSWs were predominantly local to the region, had lower income and fewer dependents, had worked as a sex worker for longer, reported more drug use, and had higher levels of STIs and HIV positivity than registered FSWs. ${ }^{13}$ The authors proposed that migrants to Tijuana who engaged in sex work were more likely to work in establishments, thereby facilitating registration. It is possible that in Panama, poverty, social discrimination and economic need may preclude unregistered FSWs from affording the registration fees required to work at entertainment establishments, leaving them with no other option except working on the streets.

HSV-2, forced sex and having a history of STIs were associated positively and independently with syphilis while registration was protective for syphilis. Sexual violence by clients is a barrier to condom negotiation, consequently increasing STIs and HIV risk. ${ }^{12}$ 14-16 Observational studies have shown that prevalent serological syphilis and HSV-2 infections increase the risk of HIV acquisition two- to threefold. ${ }^{17}{ }^{18}$ HSV-2 antibody prevalence and HIV incidence is higher among women who reported intimate partner violence. ${ }^{16}$

The potential threat of HIV spreading among unregistered FSWs in Panama is possible. HIV prevalence may increase in unregistered FSWs since they bear a higher burden of STIs, especially syphilis ( $>6 \%$ overall prevalence), and reported more contact with high-risk groups like MSM and injection drug users. An STI prevalence rate of $3 \%$ or more, especially for curable STIs, influences the rate of HIV transmission in populations with low HIV prevalence. ${ }^{19}$ STI cofactors have accounted for $50 \%$ or more of HIV transmission in low and high HIV-prevalence cities. ${ }^{20}$ Multipronged prevention interventions which encompass peer outreach services, HIV counselling and testing, education sessions and STI syndromic management and testing at clinics have led to a decline in HIV incidence, and HIV and STI prevalence among FSWs. ${ }^{21} 22$ The low seroprevalence of HCV indicates that injection drug use possibly is rare in the surveyed population.

The seroprevalences of HIV and STIs may have been underestimated in this survey. It is probable that sampling at venues where accessibility and the likelihood of participation was greater, excluded FSWs with higher risk. Areas in Colón and the Darien region were unapproachable for security reasons. FSWs in these areas and those fearful of authorities may be at greater risk of violence, have lower access to health services, and subsequently have higher risk for HIV and STIs.

Although HIV prevalence among FSWs in Panama was low, compared with registered FSWs, unregistered FSWs or 'clandestinas' bear a higher burden of HIV and STIs, were socioeconomically disadvantaged, reported higher frequencies of HIV risk behaviour, and higher risk for syphilis. Preliminary findings from this survey prompted the Panamanian $\mathrm{MoH}$ to open health clinics in areas easily accessible to unregistered FSWs. An assessment of obstacles to registration, and removal of these obstacles such that easy access to medical management of STIs for unregistered FSWs, such as those offered to registered FSWs, harm reduction, peer-based support and legal protections for those at risk of violence, are essential. 
Key messages

- HIV prevalence was low among female sex workers in Panama.

- Unregistered sex workers had a higher burden of HIV and sexually transmitted infections (STI).

- Four factors, HSV-2, forced sex, having a history of STls and registration status, were associated with syphilis.

- Prevention interventions for medical management of STIs among unregistered sex workers should be a priority.

Acknowledgements The members of the Panama HIV EPI Group were: Sonia Morales Miranda (Director of the HIV Unit, Universidad del Valle, Guatemala), Bulbulgul Aumakhan, Juana R Torres (Mujeres con Dignidad de Panamá), Iris Reyes de González, Wendy González, and Jeymy P Trujillo. We thank the staff of the Ministry of Health, Panama, for their assistance in recruitment, interviews and blood collection; the Faculty of Medicine at the University of Panama for providing laboratory facilities for all serological testing conducted for the survey; key informants at community-based non-governmental organisations PASMO/Panama (Pan American Social Marketing Organization), PROBIDSIDA (Pro Bienestar y Dignidad de las Personas con SIDA), and APLAFA (Asociación Panameña para la Planificación Familiar). Last, but not least, we thank the owners and management of all establishments for their full support of the survey in the numerous visits the survey staff conducted to gather information and recruit women.

Contributors Concept and design: $\mathrm{SH}, \mathrm{GA}, \mathrm{AN}, \mathrm{CB}, \mathrm{SP}, \mathrm{GP}, \mathrm{PS}$, JP; acquisition of data: $G A, A N, A B, M V$; analysis and interpretation of data: $S H$, PS; drafting of manuscript: $\mathrm{SH}$; critical revision of the manuscript for important intellectual content: GA, AN, AB, MV, SP, GP, PS, JP.

Funding This work was supported by funds from the Military Infectious Disease Research Program, Secretary of Science and Technology of the Republic of Panama, Pan American Health Organization, Global Funds, Centers for Disease Control and Prevention, Panama National Program of HIV.

Disclaimer The views expressed are those of the authors and should not be construed to represent the positions of the US Department of Defense.

Competing interests None.

Ethics approval ICGES, WRAIR.

Provenance and peer review Not commissioned; externally peer reviewed.

Open Access This is an Open Access article distributed in accordance with the Creative Commons Attribution Non Commercial (CC BY-NC 3.0) license, which permits others to distribute, remix, adapt, build upon this work non-commercially, and license their derivative works on different terms, provided the original work is properly cited and the use is non-commercial. See: http://creativecommons.org/ licenses/by-nc/3.0/

\section{REFERENCES}

1. WHO. Global summary of the HIV/AIDS epidemic, December 2010. Secondary Global summary of the HIV/AIDS epidemic, December 2010. 2010. http://www.who. int/hiv/data/en/

2. UNAIDS. Global Report: Unaids Report on the Global AIDS Epidemic 2010. 2011. http://www.unaids.org/globalreport/Global report.htm (accessed 23 Nov 2011).

3. Rehle T, Lazzari S, Dallabetta G, et al. Second-generation HIV surveillance: better data for decision-making. Bull World Health Organ 2004;82:121-7. doi: S0042-96862004000200009 (pii)(published Online First: Epub Date).

4. Goodman H. The antivenereal disease campaign in Panama. J Soc Hyg 1923; IX:160-67.
5. Bronfman MN, Leyva R, Negroni MJ, et al. Mobile populations and HIV/AIDS in Central America and Mexico: research for action. Aids 2002;16(Suppl 3):S42-9.

6. U.S. Department of State. 2009 Country Reports on Human Rights Practices: Panama. 2009. http://www.state.gov///dr//rls/hrrpt/2009/wha/136121.htm laccessed 30 Jan 2012).

7. Epidemiological fact sheets on HIV/AIDS and sexually transmitted infections, Panama, 2004 update. 2004. http://data.unaids.org/publications/Fact-Sheets01/ (accessed 14 Dec 2011).

8. Soto $\mathbf{R J}$, Ghee $A E$, Nunez $C A$, et al. Sentinel surveillance of sexually transmitted infections/HIV and risk behaviors in vulnerable populations in 5 Central American countries. J Acquir Immune Defic Syndr 2007;46:101-11.

9. Semaan S, Lauby J, Liebman J. Street and network sampling in evaluation studies of HIV risk-reduction interventions. AIDS Rev 2002;4:213-23.

10. Pan American Health Organization. Norms and Standards in Epidemiology: Case definition-Syphilis. Epidemiol Bull Pan Am Health Organ 2000; 21. (accessed 19 Jun 2012).

11. Aran-Matero D, Amico P, Aran-Fernandez C, et al. Levels of spending and resource allocation to HIV programs and services in Latin America and the Caribbean. PLoS One 2011;6:e22373. doi: 10.1371/journal.pone.0022373. PONE-D-11-04728 (pii) (published Online First: Epub Date).

12. Swain SN, Saggurti N, Battala M, et al. Experience of violence and adverse reproductive health outcomes, HIV risks among mobile female sex workers in India. BMC Public Health 2011;11:357. doi: 1471-2458-11-357 (pii). 10.1186/ 1471-2458-11-357(published Online First: Epub Date).

13. Sirotin N, Strathdee SA, Lozada R, et al. A comparison of registered and unregistered female sex workers in Tijuana, Mexico. Public Health Rep 2010;125 (Suppl 4):101-9.

14. Church S, Henderson M, Barnard M, et al. Violence by clients towards female prostitutes in different work settings: questionnaire survey. BMJ 2001;322:524-5.

15. Shannon K, Strathdee SA, Shoveller J, et al. Structural and environmental barriers to condom use negotiation with clients among female sex workers: implications for HIV-prevention strategies and policy. Am J Public Health 2009;99:659-65. doi:AJPH. 2007.129858 (pii). 10.2105/AJPH.2007.129858(published Online First: Epub Date).

16. Jewkes RK, Dunkle K, Nduna M, et al. Intimate partner violence, relationship power inequity, and incidence of HIV infection in young women in South Africa: a cohort study. Lancet 2010;376:41-8. doi: S0140-6736(10)60548-X (pii). 10.1016/ S0140-6736(10)60548-X(published Online First: Epub Date).

17. Ruzagira E, Wandiembe S, Abaasa A, et al. HIV incidence and risk factors for acquisition in HIV discordant couples in Masaka, Uganda: an HIV vaccine preparedness study. PLOS One 2011;6:e24037. doi: 10.1371/journal.pone.0024037. PONE-D-11-04004 (pii)(published Online First: Epub Date).

18. Barnabas RV, Wasserheit JN, Huang $\mathrm{Y}$, et al. Impact of herpes simplex virus type 2 on HIV-1 acquisition and progression in an HIV vaccine trial (the Step study). $J$ Acquir Immune Defic Syndr 2011;57:238-44. doi:10.1097/0Al.0b013e31821acb5. 00126334-201107010-00010 (pii)(published Online First: Epub Date).

19. WHO. The pre-surveillance assessment: guidelines for planning serosurveillance of HIV, prevalence of sexually transmitted infections and the behavioural components of second generation surveillance of HIV/UNAIDS/WHO Working Group on Global HIV/AIDS and STI Surveillance. 2005. http://www.who.int/hiv/pub/surveillance/sti/en/ index.html

20. White RG, Orroth KK, Glynn JR, et al. Treating curable sexually transmitted infections to prevent HIV in Africa: still an effective control strategy? J Acquir Immune Defic Syndr 2008;47:346-53. doi: 10.1097/0Al.0b013e318160d56a (published Online First: Epub Date).

21. Riedner G, Hoffmann O, Rusizoka $\mathrm{M}$, et al. Decline in sexually transmitted infection prevalence and HIV incidence in female barworkers attending prevention and care services in Mbeya Region, Tanzania. AIDS 2006;20:609-15. doi: 10.1097/01.aids. 0000210616.90954.47. 00002030-200602280-00015 (pii)(published Online First: Epub Date).

22. Ramesh BM, Beattie TS, Shajy I, et al. Changes in risk behaviours and prevalence of sexually transmitted infections following HIV preventive interventions among female sex workers in five districts in Karnataka state, south India. Sex Transm Infect 2010;86(Suppl 1):i17-24. doi: 86/Suppl 1/117 (pii) 10.1136/sti.2009.038513 (published Online First: Epub Date). 